\title{
Analyzing the Surface Properties of Organozeolite /Poly(Vinyl Chloride) Composite
}

\author{
Ceyda Bilgiç
}

\begin{abstract}
Polymer/clay nanocomposites are a class of hybrid materials composed of organic polymer matrix in which inorganic particles with nanoscale dimension are embodied. The natural zeolite (clinoptilolite) of Gördes-Manisa (in Western Anatolia of Turkey) used in this work. The O-zeolite was obtained through the purification of zeolite and was organically modified by hexadecyltrimethyl ammonium bromide (HTAB) with certain surfactant concentration. Poly(vinyl chloride)/O-zeolite composites were prepared using the solution blending method with the application of ultrasound and using trichloromethane as solvent. Ultrasonic waves were used to enhance the nanoscale dispersion of the silicate. Polymer composite of a poly(vinyl chloride) (PVC) matrix containing $5 \%$ Organozeolite $(\mathrm{OZ})$ by mass was investigated using X-ray diffraction (XRD) Scanning electron microscopy (SEM) and transmission electron microscopy (TEM). Polymer nanocomposites of a PVC matrix containing 5\% organozeolite (OZ) by mass was investigated using inverse gas chromatography (IGC). IGC was applied to characterize the surface of PVC/OZ composite.
\end{abstract}

Keywords-Poly(vinyl chloride), Nanocomposites, IGC, TEM, SEM, XRD, Characterization.

\section{INTRODUCTION}

The synthesis and development of polymer/silicate composites have attracted considerable attention from both basic research and commercial applications, as they often exhibit remarkable improvements in the mechanical properties, thermal stability, gas barrier properties, enhanced ionic conductivity, reduced flammability and biodegradation, etc., as compared with the pure polymers or conventional micro- and macro-composites [1,2]. These improvements in the properties are the result of the nanometer scale dispersion of silicate in the polymer matrix. The dispersion of zeolite layers in a monomer or polymer matrix can result in the formation of three types of composite materials. The first type is a intercalated, that can be formed if the interlayer distance increases but the layer morphology remains unchanged, an intercalated nanocomposite structure is obtained. The other type is intercalated-and-flocculated conceptually the same as

Ceyda Bilgiç, Department of Chemical Engineering, Faculty of Engineering and Architecture, Eskişehir Osmangazi University, 26480 Eskişehir, Turkey intercalated nanocomposites. However, silicate layers are sometimes flocculated due to hydroxylated edge-edge interaction of the silicate layer. The last type is the exfoliated structure, which can only be obtained if the silicate layers totally disperse in the polymer matrix. Exfoliated polymersilicate nanocomposites are especially desirable for improved properties because of the homogeneous dispersion of clay and huge interfacial area between polymer and silicate $[3,4]$.

Poly(vinyl chloride) (PVC), as an important commercial polymer, has been studied and used widely in industrial fields for many years. However, due to its inherent disadvantages, such as low thermal stability and brittleness, PVC and its composites are subject to some limitations in certain applications [5]. Therefore, it is necessary to develop new PVC products with altered properties in order broaden PVC applications. Recently, the development of PVC/layered silicate nanocomposites presents a new way to prepare high performance PVC composites. Wang et al., 2001; Wang et al., 2002, [6,7] reported the preparation and characterization of PVC-clay nanocomposites formed by both melt and solution blending. The effects of the clay loading level, the presence and amount of plasticizer, melt blending time and annealing time, etc., on the structure of the nanocomposite and the thermal and mechanical properties have also been investigated. Trillica et al., 2001 [8], took dioctylphthalate (DOP) as cointercalater for organic montmorillonite (MMT) and PVC because they found alkylammonium salts between the interlayers of organic MMT, could catalyze PVC degradation. Although DOP prevented the degradation of PVC, the MMT only acted as a plasticizer carrier and the mechanical properties of the composites were not enhanced significantly. Du et al., 2003, [9] reported more information concerning the thermal degradation and charring of PVC/MMT nanocomposite in the presence of DOP by the use of X-ray photoelectron spectroscopy and the acquisition of the carbon $(\mathrm{C} 1 \mathrm{~s})$, chlorine $(\mathrm{Cl} 2 \mathrm{p})$, and oxygen $(\mathrm{O} 1 \mathrm{~s})$ spectra. For PVCclay nanocomposites the surface at high temperatures is dominated by carbon, and not the oxygen of the clay. The presence of the clay does retard the chain-stripping degradation of the PVC and the enhanced char formation accounts for the observation of enrichment of carbon. Wan at al., 2003, [10] investigated the effect of silicate modification and MMT content on the morphology development, relaxation behavior, optical clarity and mechanical properties of the PVC/MMT nanocomposites. Despite the widespread use of the clays for preparations of composites, has not done much work on zeolites in the literature. 
In the present work, Polymer nanocomposites of a PVC matrix containing $5 \%$ organozeolite $(\mathrm{OZ})$ by mass were prepared using the solution blending method with sonication. Hexadecyl trimethyl ammonium bromide was used to modify the montmorillonite after its surface was saturated with $\mathrm{Na}^{+}$ ions. X-ray diffraction and transmission electron microscopy revealed the mixed morphology of composites. The majority of O-Zeolite is dispersed in the polymer matrix in the form of an ordered tactoid (multilayer particles) structure consisting of few silicate layers and intercalated-and-flocculated structure was achieved. Characterization studies were performed by use of XRD, TEM, SEM and IGC. Inverse gas chromatography (IGC) method at infinite dilution is applied for dispersive component of the surface free energy $\left(\gamma_{S}^{d}\right)$ of PVC nanocomposites containing 5\% wt. OZ.

\section{MATERIALS AND MethodS}

PVC was provided by Sigma-Aldrich. Hexadecyltrimethylammonium bromide (HTAB, $\mathrm{C}_{19} \mathrm{H}_{42} \mathrm{BrN}$, Purity $98 \%$ ) and sodium chloride $(\mathrm{NaCl}$, Purity $99.5 \%)$ were provided by Merck and used as received. Tetrahydrofuran (THF, Purity 99.8\%) was purchased from Sigma-Aldrich. The natural zeolite (clinoptilolite) of Gördes-Manisa (in Western Anatolia of Turkey) used in this work was supplied from Rota Mining Corporation. Because it was received as it was taken out of the ground, without having been processed, it contained many impurities. In order to purify it and also increase its zeolite content, a decantation process was applied. Following this process, the clay had been dried in an oven at $90{ }^{\circ} \mathrm{C}$ and it was ground.

\section{A. Preparation of organo-zeolite and composite}

In order to prepare the organic-intercalated zeolite firstly, 11 of $2 \mathrm{M} \mathrm{NaCl}$ solution was added to $20 \mathrm{~g}$ of purified clay and was mixed at 500rpm with a Heidolph magnetic stirrer for $24 \mathrm{~h}$ at room temperature. Then the mixture was centrifuged and rinsed three times with distilled water. The obtained Na-zeolite was dried at $110{ }^{\circ} \mathrm{C}$. Na- zeolite aqueous suspension and a certain amount of HTAB were mixed for $24 \mathrm{~h}$ by adding distilled water heated at $60{ }^{\circ} \mathrm{C}$. Next, th e mixture was centrifuged and the obtained white solid was dried in an oven after being rinsed with distilled water. After being ground, it was sieved to pass through a $90 \mu \mathrm{m}$ sieve.

Organo zeolite /PVC nanocomposites were prepared by the solution blending method. The organo zeolite was dissolved in tetrahydrofuran (THF) and the solution was magnetically stirred until all the clay appeared to be completely dispersed at $50{ }^{\circ} \mathrm{C}$. After stirring, the mixture was placed in an ultrasonic bath for sonication at room temperature, for $1 \mathrm{~h}$. PVC was dissolved in toluene at room temperature. Zeolite solution was added slowly to PVC solution and then it was stirred until the solution became homogeneous. O-zeolite/PVC mixture was placed in an ultrasonic bath, for $20 \mathrm{~min}$. After sonication it was poured in glass moulds and toluene was then evaporated which was performed by two consecutive steps: air drying for $12 \mathrm{~h}$, and in an oven at $60{ }^{\circ} \mathrm{C}$, for $3 \mathrm{~h}$. For comparison, polystyrene was dissolved in toluene, then sonicated and dried using the same procedure described above, obviously without adding Ozeolite. Finally, samples were cryo-milled using a Retsch cryogenic jar mill. The samples were labelled to describe the amount of OZ and PVC 5 mean, PVC contained and 5\% wt. of $\mathrm{OZ}$, respectively.

\section{B. Characterization techniques}

The XRD patterns were recorded between $5^{\circ}$ and $20^{\circ}(2 \theta)$ at a scanning speed of $0.02^{\circ} \mathrm{min}^{-1}$ using a Rigaku Ultima-IV diffractometer (Akishima, Japan) with $\mathrm{CuK} \alpha$ radiation (40 $\mathrm{kV} / 30 \mathrm{~mA}$ ). Scanning electron microscopy (SEM) observation of the sample was done by a Jeol JSM-5600 LV (Tokyo, Japan). Prior to SEM observation, sample was coated with a thin layer of gold using a Polaron SC7620 (Quorum Technologies Ltd., East Sussex, UK). For transmission electron microscopy (TEM), a single drop of dilute solution of sample was deposited on a copper TEM grid and dried overnight at room temperature, and examined by a Jeol JEM1220 Electron Microscopy (Tokyo, Japan, at an accelerating voltage of $80 \mathrm{kV}$ ).

The chromatographic experiments were performed with Agilent 7890 gas chromatography equipped with a flame ionization detector (FID). Nitrogen with high purity was used as the carrier gas with a flow rate of $40 \mathrm{ml} \mathrm{min}^{-1}$. IGC measurements were carried out in the temperature range of 30$60{ }^{\circ} \mathrm{C}$. About $3 \mathrm{~g}$ of sample particles of $150-200 \mu \mathrm{m}$ was filled into the stainless steel column ( $2.00 \mathrm{~m}$ long, $5.35 \mathrm{~mm}$ I.D.) via the aid of vacuum. Stainless steel column washed with methanol and acetone before packing with powders. The adsorbents were conditioned at the highest temperatures in the nitrogen gas flow for $4 \mathrm{~h}$ before the measurements. Methane is used for determining the dead volume. At least four determinations were used in averaging the net retention volume $\left(\mathrm{V}_{\mathrm{N}}\right)$.

\section{IGC theory}

Inverse gas chromatography (IGC) is a derivation of conventional gas chromatography. However, unlike analytical chromatography, the material being investigated is the solid in the gas chromatography column. The retention volume of the mobile phase (probe) indicates the interaction between the probe and the surface of the material in the column [11]. IGC provides an excellent method to measure the surface energy of rough and porous powders. The surface energy is the result of the unbalanced molecular forces at the surface of the solid. It can be considered to be formed by two different contributions: dispersive and specific. The retention time of a series of homologous $n$-alkanes is used to determine the dispersive component of the surface free energy $\left(\gamma_{S}^{\mathrm{d}}\right)$ of PVC/OZ. In IGC literature, $\gamma_{S}^{\mathrm{d}}$ is commonly determined from the following equation, which was introduced by Lavielle and Schultz: 


$$
R T \operatorname{Ln} V_{N}=2 N \cdot\left(\gamma_{S}^{d}\right)^{1 / 2} \cdot a \cdot\left(\gamma_{L}^{d}\right)^{1 / 2}+\mathrm{C}
$$

Here, $R$ is the gas constant, $T$ is the absolute column temperature, $a$ is the molecular surface area coated with a kind of adsorbed alkane, $N$ is the Avogadro's number, $\gamma_{L}^{d}$ is the dispersive component of the surface free energy of the probe, $C$ is a constant, and $V_{N}$ is the net retention volume of the $n$ alkane probe. The net retention volume $\left(V_{N}\right)$ is calculated using the equation below [12]:

$$
\mathrm{V}_{N}=\mathrm{F}_{\mathrm{o}} \frac{\mathrm{T}}{\mathrm{T}_{\mathrm{o}}} \frac{3}{2}\left[\frac{\left(\mathrm{P}_{i} / \mathrm{P}_{\mathrm{o}}\right)^{2}-1}{\left(\mathrm{P}_{i} / \mathrm{P}_{o}\right)^{3}-1}\right]\left(\mathrm{t}_{\mathrm{A}}-\mathrm{t}_{0}\right)
$$

Here, $t_{A}$ is the retention time of the probe, while $t_{0}$ is the retention time of the probe which has no interaction with the solid in the column (marker). $P_{i}$ and $P_{o}$ are, respectively, the inlet and outlet pressures of the carrier gas, while the $T / T_{o}$ ratio is used in order to get the value of the flow rate at the column temperature $(T)$ from the measurement of the flow rate at ambient temperature $\left(T_{o}\right)$. The flow rate of the carrier gas which is measured at the column outlet and at ambient temperature is expressed as $F_{o}$.

\section{RESULTS AND DISCUSSION}

The adsorption runs were performed at infinite dilution conditions. The chromatographic peaks of $n$-alkanes on $\mathrm{PVC/OZ}$ were symmetrical. So retention time is independent of the amount of injected. The value of $\gamma_{S}^{\mathrm{d}}$ represents the interaction of the surface with $n$-alkanes and hence is a measure of how easily the surface can polarize the probe [13]. The dispersive component of the surface free energy, $\gamma_{S}^{d}$, was determined by injection of a homologous series of $n$-alkanes having between 8 and 10 carbon atoms. One of the most commonly measured parameters for the description of the energy situation on the surface of a solid is the surface energy. The surface energy can affect, e.g. catalytic activity or the strength of particle-particle interaction. The dispersive components of $\mathrm{PVC} / \mathrm{OZ}$ at experimental temperatures were calculated from Equation-1. Plotting $R T \ln V_{N}$ against $a \cdot\left(\gamma_{L}^{d}\right)^{1 / 2}$ yields a straight line with the slope of $\gamma_{S}^{\mathrm{d}}$, which can be seen for $\mathrm{PVC} / \mathrm{OZ}$ in Figure 1. Table 1 shows the dispersive component of the surface free energy, $\gamma_{S}^{\mathrm{d}}$, of PVC/OZ. It was observed that $\gamma_{S}^{\mathrm{d}}$ values decrease with increasing temperature.

TABLE I

VALUES OF DisPersive COMPONENT OF THE SURFACE ENERGY MEASURED ON 5\% OZ-PVC AT VARIOUS TEMPERATURES

\begin{tabular}{|l|c|c|c|c|}
\hline $\mathrm{T}\left({ }^{\circ} \mathrm{C}\right)$ & 30 & 40 & 50 & 60 \\
\hline$\gamma_{S}^{\mathrm{d}}\left(\mathrm{mJ} / \mathrm{m}^{2}\right)$ & 27,264 & 26,478 & 22,816 & 17,825 \\
\hline
\end{tabular}

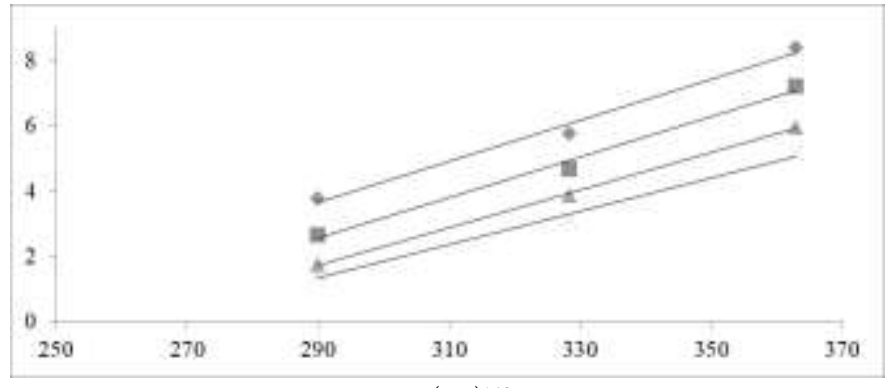

Fig. 1 The $R T \ln \left(V_{N}\right)-a \cdot\left(\gamma_{L}^{d}\right)^{1 / 2}$ graphs for $5 \%$ OZ-PVC

Complementary to XRD, TEM is the most popularly employed technique to determine composite morphology, using TEM one can image the composite structure. In general, one collects several images at high and low magnification and at several positions in the nanocomposite sample. Both a low magnification image, to show the global dispersion of the additives in the polymer, and a higher magnification image, to evaluate the registry of additives are needed. The ordered intercalates exhibit microstructures very similar to the unintercalated organically modified layered silicate. Polymer intercalation occurs as a front which penetrates the primary organically modified layered silicate particle from the external edge. The disordered composites exhibit heterogeneous microstructures with increased layer disorder and spacing towards the polymer-primary particle boundary. In these hybrids, individual silicate layers are observed near the edge whereas small coherent layer packets separated by polymerfilled gaps are prevalent toward the interior of the primary particle. The heterogeneous microstructure indicates that the formation of these disordered composites occurs by a more complex process than simple sequential separation of individual layers starting from the surface of the crystallites and primary particles. In general, the features of the local microstructure from TEM give useful detail to the overall picture that can be drawn from the XRD results and enhance the understanding of various thermodynamic and kinetic issues surrounding composite formation $[14,15]$.

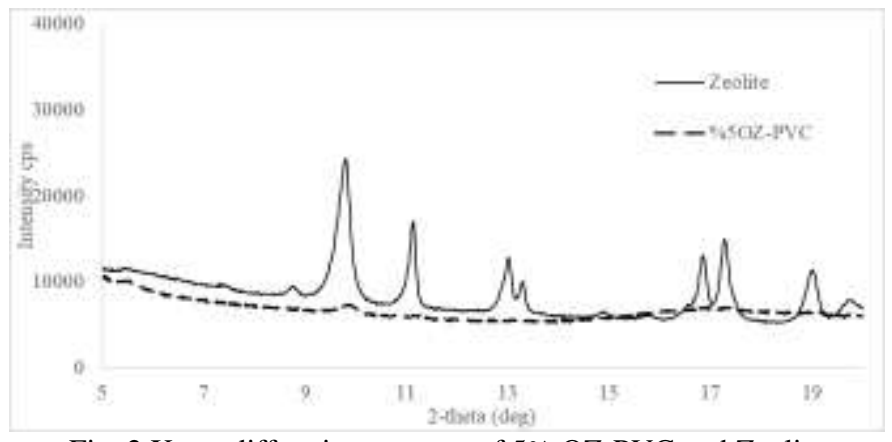

Fig. 2 X-ray diffraction patterns of 5\% OZ-PVC and Zeolite

It is well known that only materials ordered enough to diffract X-ray can be detected; disordered materials will show no pattern with the X-ray technique. Generally, the formation of an intercalated nanocomposite results in an increase in basal 
spacing in the XRD pattern, while the formation of an exfoliated nanocomposite leads to the complete loss of registry between the layers and therefore no peak can be observed [14]. Figure 2 shows the XRD patterns of zeolite, and PVC/Ozeolite. Dispersion of the zeolite particles in the polymer matrix is very important because the filler dispersion has a main effect on the final morphology and properties of the polymer nanocomposites. In study, the XRD peak of PVC-OZ composites appeared at the $2 \theta$. However, the OZ/PVC composites, prepared using $5 \%$ wt of OZ, did not showed any noticeable diffraction peak at the $2 \theta$ values from 1 to $20^{\circ}$. This result suggests that the zeolite platelets was able to intercalated and-flocculated dispersed in the PVC matrix. Because the peak intensity is very low; indicating that an insignificant amount of agglomeration was present, probably due to zeolite platelet reorganization during ring-opening polymerization in the absence of shear flow. The XRD patterns suggest that the strongly hydrophobic PVC was inserted into the galleries of the hydrophilic zeolite through emulsion polymerization $[16,17]$.

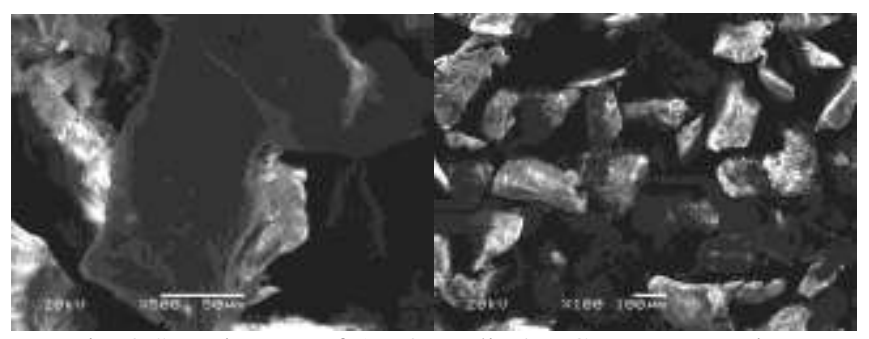

Fig. 3 SEM images of 5\% O-zeolite/PVC nanocomposites at magnifications of a) $\times 100$ and b) $\times 500$

The morphology of the composites is evaluated by using SEM and TEM. The images of SEM give information about the reinforcement aggregates, adhesive failures and phase boundaries in composites, surface roughness, and fractured surfaces. The roughness like recesses and protrusions on the surface is very important because it intensively affects the wettability property. This property is known as the tendency of fluids to stick to the surface and can be determined by the characteristics of the surface structure $[18,19]$. In this study, SEM analysis is performed to display the surface of materials and the distribution of reinforcement in polymer matrix. The SEM images of composites are given at magnifications of $\times 100$ and $\times 500$ in Figure 3. SEM images of composite exhibit quite well dispersion. Some bridges within the polymer matrix and dangling structures are also observed. These results may be attributed to the good dispersion of the zeolite and the compounding ratio seems not to be a prominent parameter within this phenomena.

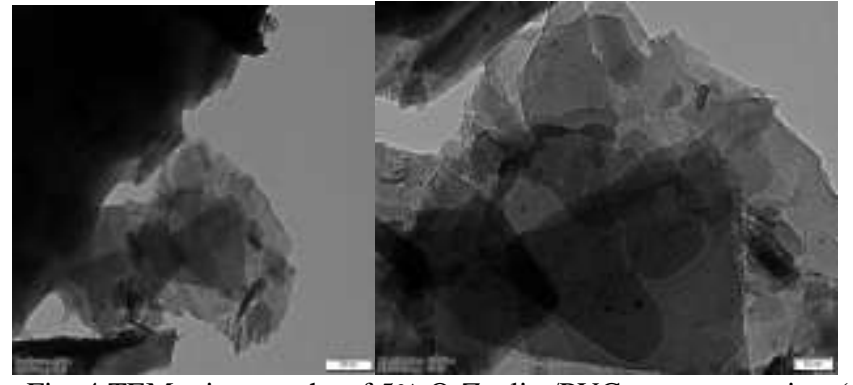

Fig. 4 TEM micrographs of 5\% O-Zeolite/PVC nanocomposites (a) at low magnification (b) at higher magnification

The dispersion microstructure of the intercalated andflocculated zeolite layers was also examined by means of TEM. Direct evidence for the dispersion of the O-zeolite in the final polymer composites can be obtained from TEM. The intercalated and flocculated trend of the zeolite in the PVC/zeolite nanocomposites was confirmed by TEM, as shown in Figure 4. Figure 4 shows the TEM image of 5\% Ozeolite/PVC, which demonstrates that the zeolite layers are structured in good order and are well, dispersed in the polymer matrix $[20,21]$. The image confirms that the synthesized composite is a nanoscale material and no delamination of the O-zeolite layers took place during intercalation.

\section{CONCLUSION}

The composite's surface usually has different chemical and physical properties from both matrix and filler, probably due to changing surface roughness and chemical heterogeneity. It is important to understand the zeolite-filled polymer surfaces to control adhesion, wettability, and printability properties of these nanocomposites. In this study, O-zeolite/PVC composites were prepared by solution blending method using TCM as solvent. Solution blending is widely used in composite preparation. It is a simple way to obtain silicate/polymer composites if both the polymer and the nanoparticles are dissolved or dispersed in solution. The toxic solvent in the process could be recycled. In solution blending method, it is easy to control the concentrations of the polymer and inorganic components. In the present work, ultrasonication is applied to further improve the exfoliation of the O-zeolite.

It is important to understand the zeolite-filled polymer surfaces to control adhesion, wettability, and printability properties of these composites. IGC is a very sensitive and reliable technique for distinguishing nanocomposite surfaces. IGC at infinite dilution was used to find how PVC/OZ would change the dispersive component of the composite. The experimental results indicate that $\gamma_{S}^{\mathrm{d}}$ values of studied materials gradually decreased with increasing column temperature which is consistent with the fundamental concept of Gibbs free energy. A combination of XRD, SEM and TEM studies showed that the nanocomposites obtained have a mixed nanomorphology. From SEM, TEM and XRD results, the PVC was intercalated and-flocculated into the zeolite layers, and had fine dispersion in the PVC matrix. HTAB-zeolite in the 
PVC/O-zeolite nanocomposite indicates that the zeolite was transformed into fine particles and dispersed homogeneously in the PVC matrix after polymerization. SEM, TEM and XRD analyses provided a high depth of focus to the morphology and the quite well dispersions are observed. This will provide the necessary flexibility for the potential applications of these new composites.

\section{ACKNOWLEDGMENT}

This study is the part of a project (2017-1426) supported by the Research Fund of Eskişehir Osmangazi University.

\section{REFERENCES}

[1] F. Annabi-Bergaya, Layered clay minerals. Basic research and innovative composite applications, 2008, Microporous Mesoporous Material, vol:107, pp:141-8.

[2] S. Pavlidou, C.D. Papaspyrides, A review on polymer-layered silicate nanocom-posites. Progress Polymer Science, 2008, vol: 33, pp. 111998.

[3] J. Zhang, D.D. Jiang, D. Wang, C.A. Wilkie, Styrenic polymer nanocomposites based on an oligomerically-modified clay with high inorganic content, Polymer Degradation and Stability, 2006, vol:91, pp:2665-2674.

[4] L.B. de Pavia, A.R. Morales, F.R.V. Díaz, Organoclays: Properties, preparation and applications. Applied Clay Science, 2008, vol:42, pp:824.

[5] B. Dietrich, 2001, PVC -Origin, Growth, J. Vinyl and Addit. Technol., 2001, vol:7(4), pp:168-176.

[6] D. Wang, D. Parlow, Q. Yao, C.A. Wilkie, PVC-Clay Nanocomposites: Preparation, Thermal and Mechanical Properties, J. Vinyl and Addit. Technol., 2001, vol:7, pp:203-213.

[7] D. Wang, D. Parlow, Q. Yao, C.A. Wilkie, Melt Blending Preparation of PVC-Sodium Clay Nanocomposites, J. Vinyl and Addit. Technol., 2002, vol:8, pp:139.

[8] J. Trillica, A. Kalendova, Z. Malac, J. Simonik, in: Proc. of SPE ANTEC, Dallas, Texas, May 6-10, 2001, p. 2162.

[9] J. Du, D. Wang, C.A. Wilkie, J. Wang, An XPS investigation of thermal degradation and charring on poly(vinyl chloride)-clay nanocomposites, Journal Polymer Degradation and Stability, 2003, vol:79, pp. 319-324.

[10] C. Wan, X. Qiao, Y. Zhang, Y.X. Zhang, Effect of different clay treatment on morphology and mechanical properties of PVC-clay nanocomposites, Polymer Testing,2003, vol: 22, pp. 446-453.

[11] N. Cordeiro, C. Gouveia, M.J. John, Investigation of Surface Properties of Physico-Chemically Modified Natural Fibres Using Inverse Gas Chromatography, Industrial Crops and Products, 2011, vo: 133, pp:108115.

[12] F. Thielmann, Introduction into the characterization of porous materials by inverse gas chromatography, J. Chromatogr. A., 2004, vol: 1037, pp:115-123.

[13] G.J. Price, D.M. Ansari, An inverse gas chromatography study of calcination and surface modification of kaolinite clays. Phys. Chem. Chem. Phys., 2003, vol:5, pp.5552-5557.

[14] B. Pourabas, V. Raeesi, 2005, Preparation of ABS/montmorillonite nanocomposite using a solvent/non-solvent method., Polymer, 2005, vol:46, pp: 5533-5540.

[15] L.J. Wang, S.P. Su, D. Chen, C.A. Wilkie, Variation of anions in layered double hydroxides: effects on dispersion and fire properties, Journal Polymer Degradation and Stability, 2009, vol: 94, pp.770-781.

[16] M.W. Noh, D.C. Lee, Synthesis and characterization of PS-clay nanocomposite by emulsion polymerization, 1999, Polymer Bulletin (Berlin), vol: 42, pp. 619-626.

[17] M. Pan, X. Shi, X. Li, H. Hu, L. Zhang, Morphology and properties of pvc/clay nanocomposites via in situ emulsion polymerization. Journal of Applied Polymer Science, 2004, vol: 94, pp: 277-86.

[18] J. Sayyad Amin, S.H. Ayatollahi, A. Alamdari, Fractal characteristics of an asphaltene deposited heterogeneous surface. Applied Surface Science, 2009, vol:256, pp: 67-75.
[19] V. Sajini, J. Paul, N. Mahanta, S. Valiyaveettil, Flexible conductive graphene/poly(vinyl chloride) composite thin films with high mechanical strength and thermal stability. Carbon, 2011, vol:49, pp:198-205.

[20] TH Kim, LW Jang, DC Lee, HJ Choi, MS Jhon, Synthesis and Rheology of Intercalated Polystyrene/ $\mathrm{Na}^{+}$-Montmorillonite Nanocomposites, Macromolecular Rapid Communications, 2002, vol: 23, pp:191-196.

[21] T. Karayıldırım, J. Yanık, M. Yuksel, M. Sağlam, C. Vasile, H. Bockhorn, The effect of some fillers on PVC degradation, Journal of Analytical and Applied Pyrolysis, 2006, vol:75, pp:112-119. 